\title{
Surface State Electrons on a Hydrogen Film. 2. Influence of Adsorbed Helium Films
}

\author{
Kimitoshi Kono \\ Institute of Physics, University of Tsukuba, Tsukuba, Japan \\ and \\ Uwe Albrecht and Paul Leiderer \\ Universität Konstanz, Konstanz, Germany
}

(Received May 16, 1991; revised August 6, 1991)

We have investigated the conductivity of surface state electrons on liquid ${ }^{4} \mathrm{He}$ films supported by a quench-condensed thin solid hydrogen substrate. A pronounced dip in the ac-conductivity was observed as the He film thickness reached about 10 layers. This phenomenon was studied at different temperatures (range between 1.6 and $2 \mathrm{~K}$ ), electron densities from 0.9 to $19 \times 10^{8} \mathrm{~cm}^{-2}$, frequencies between 1 and $100 \mathrm{kHz}$, and for various amplitudes of the ac driving voltage. Ripplon scattering and a ripplonic polaron are discussed as sources for the conductivity dip.

\section{INTRODUCTION}

In a previous paper ${ }^{1}$ (hereafter referred to as I) we have presented results for the conductivity of surface state electrons (SSE) on quenchcondensed hydrogen films. It is the purpose of the present paper to show how the electron conductivity is affected when a film of ${ }^{4} \mathrm{He}$ is adsorbed onto the hydrogen surface.

Phenomena related to the build-up of a helium film on solid hydrogen have been reported previously by Paalanen and $\mathrm{Iye}^{2}$ (hereafter referred to as PI) and later by Cieslikowski et $a l^{3} \mathrm{Up}$ to a few atomic layers the conductivity showed oscillations as a function of film thickness (provided the temperature was low enough), which were assigned to the completion of subsequent monolayers. The amplitude of the oscillations was found to decay, as the film thickness increased. Shortly before the complete condensation of a saturated helium film (with a typical thickness of several tens of $\mathrm{nm}$ ) a dip in the conductivity was observed, which, according to PI, appeared 
at a film thickness of between 3 and $10 \mathrm{~nm}$. In the following we shall focus on this dip and on its dependence upon various experimental parameters.

The appearance of a conductivity dip is not a phenomenon unique to SSE on superfluid ${ }^{4} \mathrm{He}$ films covering solid hydrogen. Qualitatively similar results have been obtained by several groups for other substrate combinations: the first observation of a conductivity dip in the transition region between thin and thick saturated ${ }^{4} \mathrm{He}$ films was reported by Andrei ${ }^{4}$ for a sapphire substrate; Kajita and Sasaki, using solid Ne as a substrate, ${ }^{5}$ also observed this phenomenon; furthermore, as shown by Cieslikowski et al., ${ }^{3}$ a dip appears not only for superfluid ${ }^{4} \mathrm{He}$, but also for normal ${ }^{3} \mathrm{He}$ films on solid $\mathrm{H}_{2}$. Although it is not clear yet whether all these observations can be attributed to the same effect, the results look strikingly similar. To pinpoint the mechanisms leading to this possibly rather general phenomenon, we have undertaken a systematic study in order to provide a set of data for quantitative theoretical considerations.

SSE on a quench-condensed hydrogen film exhibit the conductivity dip in a well-pronounced and reproducible way. We have investigated the dependences of the phenomenon on temperature (1.6-2.0 K), frequency $(1.0-100 \mathrm{kHz})$, driving voltage, electron density, and quality of the hydrogen surface. Our data show that the size of the conductivity dip depends on the quality of the hydrogen surface and on the electron density, and that the position of the dip shifts slightly with the electron density. The other parameters have had little effect on the phenomenon within the present experimental range.

The following section deals with the electron wave function of the surface state and with PI's explanation for the conductivity dip based on the electron-ripplon scattering. The experimental method will be explained in Sec. 3. As to experimental details we refer also to I. Experimental results will be presented in Sec. 4. Section 5 gives a discussion of our results in connection not only with PI's explanation, but also with a proposed polaron formation. The last section, Sec. 6 , summarizes the present work.

\section{THEORY}

The ground state of surface state electrons (SSE) on a liquid helium surface is characterized by the following hydrogen-atom-like wave function:

$$
\psi(z)=2 \gamma^{3 / 2} z e^{-\gamma z}
$$

Here the $z$-axis is perpendicular to the surface and the liquid occupies the half space $z<0$. The coefficient $\gamma$ is the inverse of the effective Bohr radius $a_{E B}$, which is $7.8 \mathrm{~nm}$ on a liquid helium and $1.7 \mathrm{~nm}$ on a solid hydrogen 
surface (due to the larger polarizability of solid $\mathrm{H}_{2}$ ). Parallel to the surface the wave function is a plane wave, because the system has translational symmetry in this direction.

In the present work, we deal with a system consisting of a thin helium film on a solid hydrogen surface. Although Eq. (1) is not the exact wave function for such a system, we may adopt the form of Eq. (1) as a trial function for the variational calculation, regarding $\gamma$ as a variational parameter. Hence as the helium film thickness increases from zero to infinity on a surface of solid hydrogen, $a_{E B}$ is to vary from 1.7 to $7.8 \mathrm{~nm}$.

On a flat hydrogen surface the electron mobility is limited by two sources, namely (electron-) helium-gas-atom scattering and (electron-) ripplon scattering. It should be mentioned here that in this case the dispersion relation of the ripplons is modified as compared to the expression for a deep liquid due to the small film thickness.

A classical expression for the mobility is given by $\mu=e \tau / m$, where $e$ and $m$ are the charge and mass of an electron, and $\tau$ is the scattering time. The scattering rate $1 / \tau$ is the sum of the helium-gas-atom and ripplon scattering rates $1 / \tau_{G}$ and $1 / \tau_{R}$, respectively, thus $1 / \tau=1 / \tau_{G}+1 / \tau_{R}$. The helium-gas-atom scattering rate amounts to ${ }^{6}$

$$
\frac{1}{\tau_{G}}=\frac{3 \pi \hbar A \gamma n_{G}}{8 m}
$$

where $A$ is the scattering cross section of a helium atom $\left(\sim 5.0 \times 10^{-16} \mathrm{~cm}^{-2}\right.$, $)$ and $n_{G}$ is the density of helium atoms in the gas phase. The ripplon contribution $1 / \tau_{R}$ is given $b^{2}$

$$
\frac{1}{\tau_{R}}=\frac{\left(e F_{z}\right)^{2}}{4 \hbar \sigma}\left(1-\sqrt{\frac{1}{1+a}}\right) \quad a=\frac{8 m k_{B} T \sigma d^{4}}{3 \alpha_{H} n_{H e} \hbar^{2}}
$$

Here, $F_{z}$ is the electric field which presses the electrons onto the helium film, $\sigma$ is the surface tension of liquid helium, $k_{B}$ the Boltzman constant, $T$ the temperature, $d$ the thickness of the helium film, $\alpha_{H}$ the van der Waals-Hamaker constant for a helium atom on solid hydrogen, and $n_{\mathrm{He}}$ is the density of liquid helium. In Eq. (3) a relatively weak contribution of the polarization term is neglected.

The electric field $F_{z}$ consists of two parts as follows:

$$
F_{z}=F_{0}+\int_{0}^{\infty} \frac{e \lambda_{2}}{(z+d)^{2}} \psi(z)^{2} d z \quad \lambda_{2}=\frac{\varepsilon_{1}\left(\varepsilon_{2}-\varepsilon_{1}\right)}{\left(\varepsilon_{1}+1\right)^{2}\left(\varepsilon_{2}+\varepsilon_{1}\right)}
$$

$\varepsilon_{1}$ and $\varepsilon_{2}$ are the dielectric constants of liquid helium (1.057) and solid hydrogen (1.29), respectively. $F_{0}$ is the externally applied electric holding 
field, which is related to the areal electron density $n_{s}$ by $F_{0}=2 \pi e n_{s}$. The second term is due to the image field from the hydrogen substrate.

One can easily see from Eqs. (3) and (4) that the scattering rate $1 / \tau_{R}$ becomes very small both for the limits of $d \rightarrow 0$ and $d \rightarrow \infty$, as long as $F_{0}$ is small. In between the rate has a maximum, or conversely the mobility has a minimum. It was the suggestion of PI to interpret the conductivity dip in this context.

\section{EXPERIMENTAL}

The surface state electrons were located on a quench condensed hydrogen film. A schematic drawing of the sample configuration is shown in Fig. 1. About $2 \mu \mathrm{m}$ of hydrogen were condensed onto a thin glass plate at a temperature of $1.5 \mathrm{~K}$, which covered the electrodes for measuring the SSE conductivity. The electrodes consisted of high-conductivity copper and were thermally anchored to the bottom of the sample cell.

The electrons were supplied by a filament heated by a short current pulse. In order to decelerate the electrons a small amount of helium gas was introduced into the cell. At low temperature the gas condensed onto the hydrogen surface forming a saturated helium film.

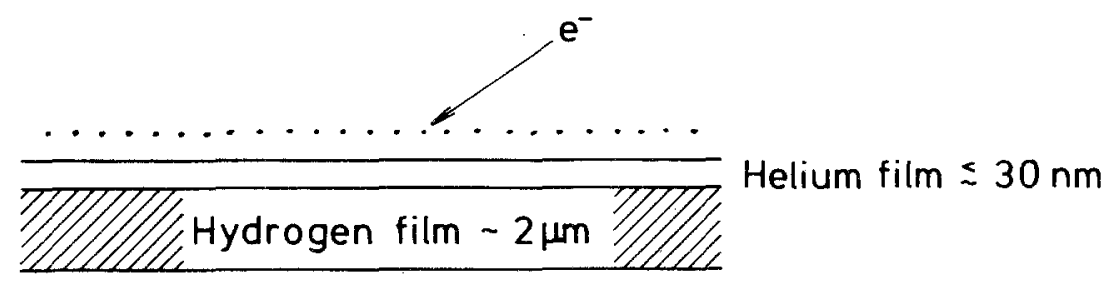

$$
\text { Glass }-0.2 \mathrm{~mm}
$$

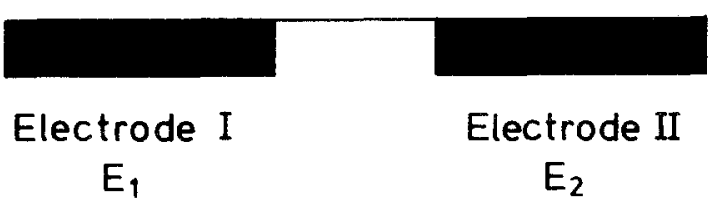

Fig. 1. Schematic drawing of the surface state electrons on a quench condensed hydrogen film whose surface is covered with a helium film. The hydrogen film is condensed on a thin glass plate under which two electrodes are attached for the conductivity measurement. 
The principle of the conductivity measurement was the so-called Sommer-Tanner ${ }^{7}$ method. The conductivity $\sigma_{\square}$ and sheet capacitance of the SSE were obtained by analyzing the in-phase and $90^{\circ}$ out-of-phase component of the SSE current, which was induced by the ac driving voltage applied to electrode $E_{2}$ and registered by electrode $E_{1}$ (Fig. 1). Both components of the signal were detected by using a two-phase lock-in amplifier and recorded on a computer. The details were described in $I$.

The thickness of the adsorbed helium film was estimated by monitoring the frequency change of a quartz oscillator. The quartz was of a tuning-fork type and operated at about $32 \mathrm{kHz}$. ${ }^{*}$ The driving circuit consisted of a standard C-MOS IC, which was immersed in liquid helium to improve the stability. It was quite stable below $4.2 \mathrm{~K}$, although a strong temperature dependence of the oscillating frequency was observed at higher temperatures.

Figure 2 shows a typical performance of the thickness gauge. In this measurement the sample cell was fed with helium at a constant rate and at

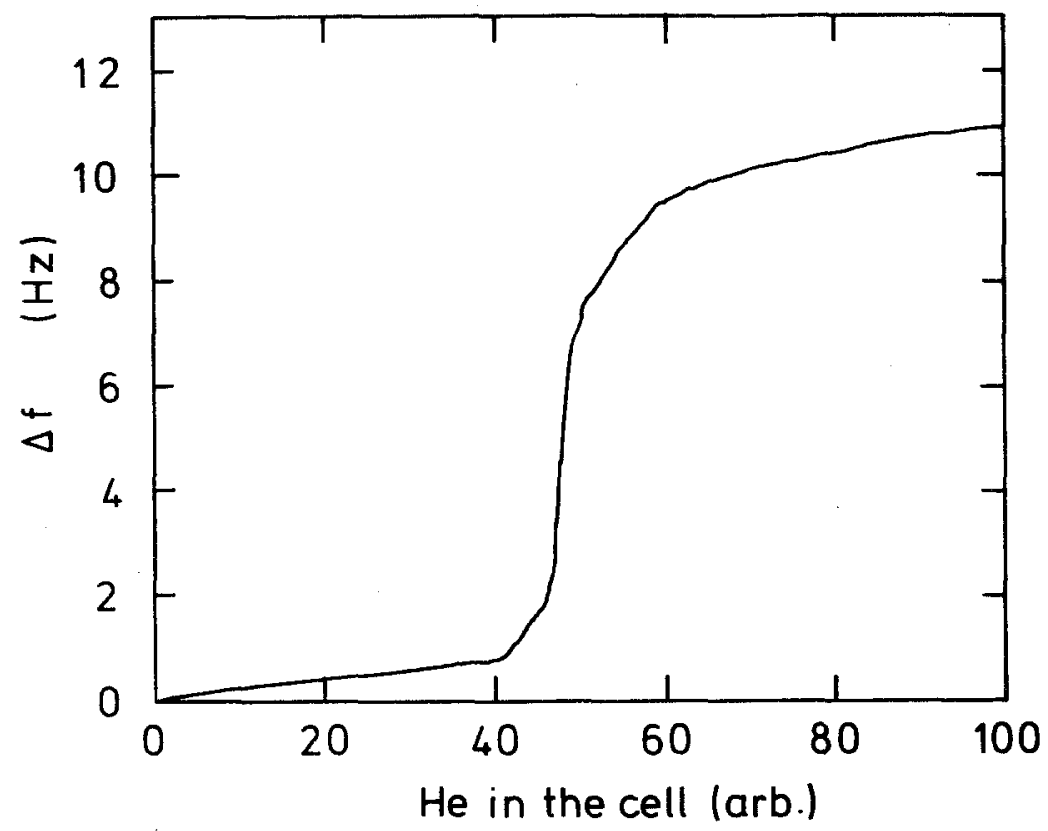

Fig. 2. Frequency change of a quartz oscillator due to helium adsorbed on the quartz. The frequency shift was measured at $1.5 \mathrm{~K}$, while helium was introduced gradually. The steep increase indicates the formation of a saturated film as given by Eq. (5).

* We are grateful to H. Kinder and L. Koester (Technical University Munich) for making us acquainted with this method. 
a fixed temperature of $1.5 \mathrm{~K}$. At first the frequency shift $|\Delta f|$ (the decrease in the oscillating frequency corresponds to the increase of the mass of the oscillator) increased rather slowly with the amount of helium admitted to the cell, followed by an abrupt rise and leveling off as the saturated vapor pressure of helium was reached in the cell. This behavior is qualitatively in agreement with the van der Waals isotherm,

$$
d^{-3} \sim T \ln \frac{P_{0}}{P}
$$

Here $P_{0}$ is the saturated vapor pressure of ${ }^{4} \mathrm{He}$ at the temperature $T$ and $P$ is the actual helium pressure in the cell. (Since the hydrogen substrate is at a height $h=1 \mathrm{~cm}$ above the cell bottom and therefore above the free liquid surface at bulk condensation, the saturated film thickness is determined by $d^{3}=\alpha_{H} / m g h$, amounting to $d=27 \mathrm{~nm}$ for a van der WaalsHamaker constant $\alpha_{H}=20 \mathrm{~K}$ (layer) ${ }^{3}$ for $\mathrm{He}$ on $\mathrm{H}_{2}{ }^{2,3}$ )

Although it readily yields qualitative results, a more accurate analysis with this thickness gauge is hampered by the fact that it measures the helium thickness on the quartz rather than on the hydrogen surface. As an approximation, however, we expect the following relation to hold between the thickness of the helium film on hydrogen, $d_{H}$, and $d_{X}$ on quartz:

$$
\frac{d_{X}}{d_{H}}=\left(\frac{\alpha_{X}}{\alpha_{H}}\right)^{1 / 3}
$$

where $\alpha_{X}$ is the van der Waals-Hamaker constant for quartz, $\alpha_{X}=25 \mathrm{~K}$ (layer) $^{3} .^{8}$

A further complication arises from the superfluidity of the helium film, which requires a calibration procedure to obtain the thickness quantitatively. We leave this problem for the future. Nevertheless the quartz gauge can be used to obtain a rough measure of the helium film thickness in the present work.

\section{EXPERIMENTAL RESULTS}

The overall temperature dependence of the SSE conductivity in our system can be characterized by three regions, as shown by the typical experimental trace shown in Fig. 3 (in this case the quench-condensed hydrogen film had been annealed at $4.2 \mathrm{~K}$ for $1 \mathrm{~h}$ ):

1. At low temperature $(<1.65 \mathrm{~K})$ the hydrogen substrate is covered by a saturated helium film. The surface roughness is sufficiently small so that $\sigma_{\square}$ is determined by scattering of the electrons from helium atoms in the gas phase. The temperature dependence of the scattering rate therefore 


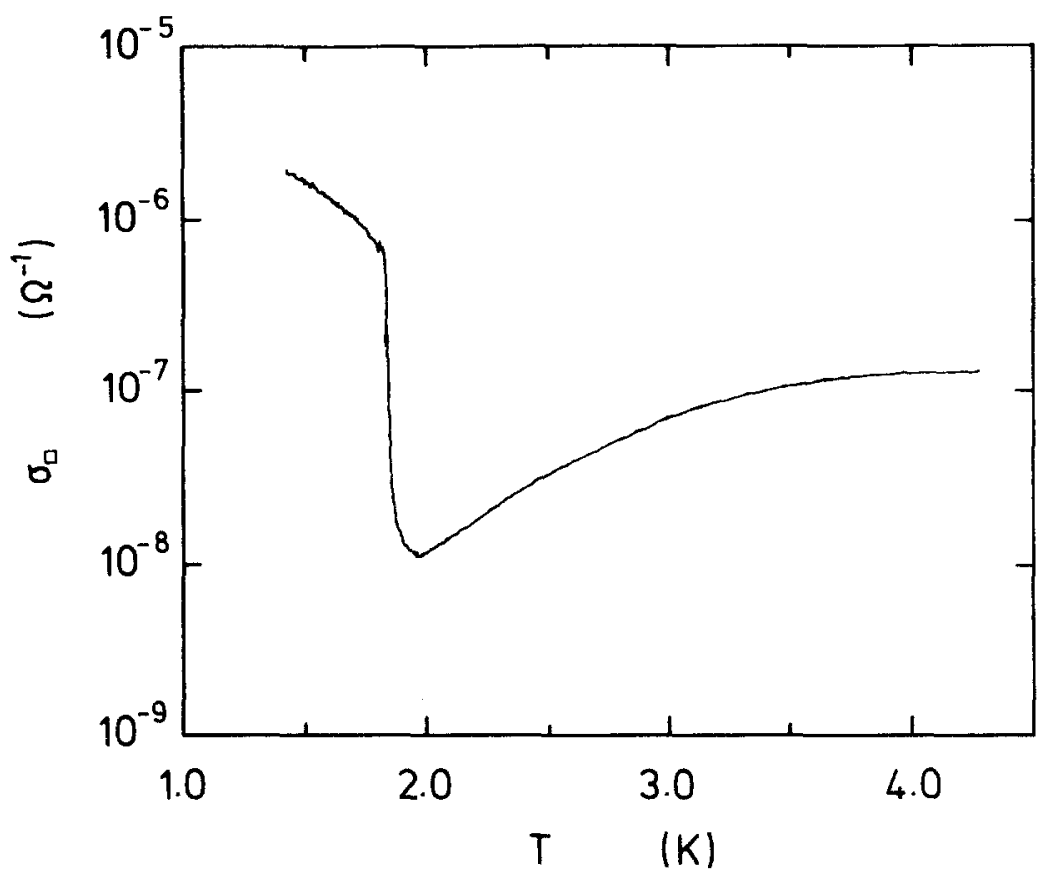

Fig. 3. Overall temperature dependence of the SSE conductivity. Before the measurement, the hydrogen had been annealed at $4.2 \mathrm{~K}$ for 1 hour. Below about $1.6 \mathrm{~K}$ the hydrogen was covered with a saturated helium film, leading to a high conductivity. This region is quite similar to that on the bulk helium, that is, the conductivity is predominated by scattering from helium gas atoms. Above $1.9 \mathrm{~K}$ the conductivity is mainly limited by the roughness of the hydrogen substrate.

follows the gas density, similar to the behavior of SSE on bulk helium in this temperature range.*

2. At high temperature $(>1.9 \mathrm{~K})$, where the helium film has essentially evaporated (apart from the very first monolayers which are more strongly bound), the electron conductivity is governed mainly by the surface roughness of the solid substrate. The temperature dependence of the conductivity can be described by a thermally activated process in this regime with an activation energy $E_{c}$ depending on the annealing treatment of the hydrogen (see I). $E_{c}$ gives a measure of the surface roughness, as long as the electron density $n_{s}$ is kept constant.

3. In the intermediate region between 1.65 and $1.9 \mathrm{~K}$ a sharp drop in the conductivity is observed, reflecting the transition between a saturated and an extremely thin helium film.

* On bulk helium, the scattering from ripplons is important only below $1 \mathrm{~K}$. At higher temperature, scattering from gas atoms dominates. 


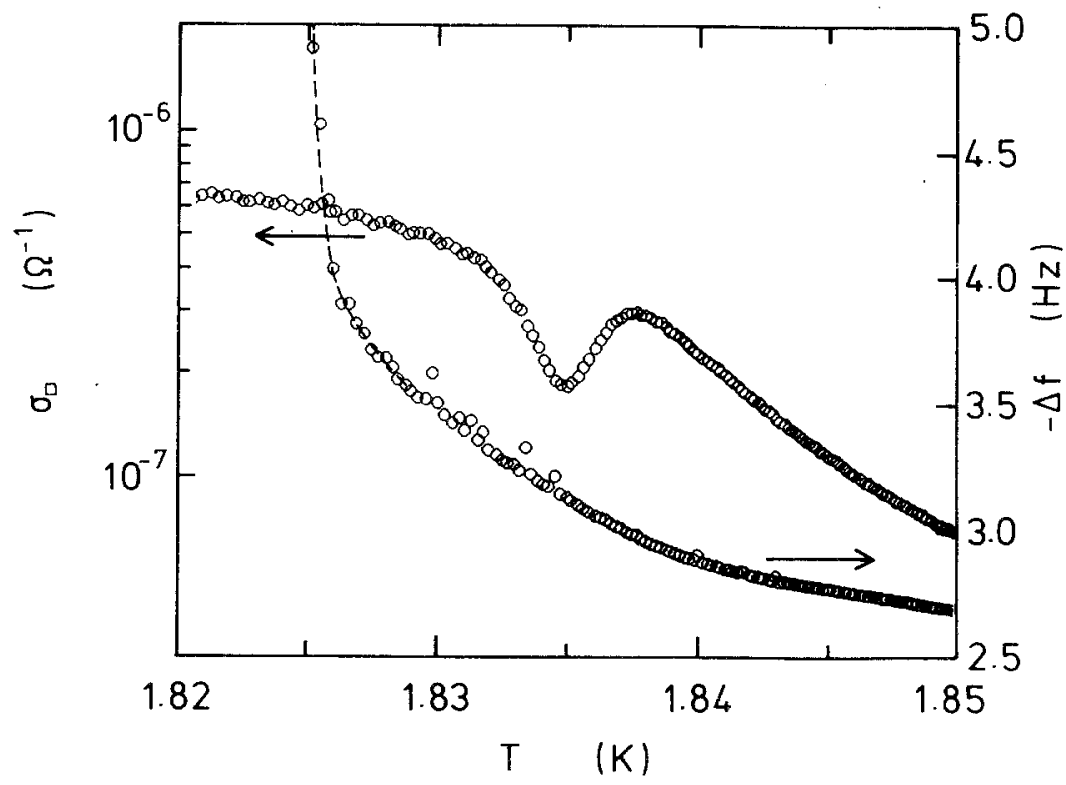

Fig. 4. Expanded view of the data of Fig. 3 in the vicinity of the conductivity dip, together with the frequency change of the quartz oscillator which was used to monitor the helium thickness.

The conductivity dip, which is the main topic here, is hardly discernible on the displayed temperature scale. We therefore show this region on an expanded scale in Fig. 4, together with the frequency change $\Delta f$ of the quartz thickness gauge. $\Delta f$ is seen to vary monotonously with temperature in the displayed interval, indicating that the film thickness changes smoothly in the regime of the dip. From a rough calibration according to Fig. 2 we obtain a film thickness of about 10 atomic layers at the position of the dip minimum. This is consistent with PI's experimental result of 3-10 nm for the appearance of the dip.

In the following we consider the influence of various parameters on the conductivity dip, like the substrate roughness, the amount of helium in the sample cell, SSE density, and amplitude and frequency of the ac driving field of the conductivity measurement. We mention here that the dip did not display any hysteresis on warming or cooling within our temperature resolution.

\subsection{Surface Roughness}

Figure 5 shows the conductivity for three different annealing stages. The other parameters, in particular the electron density and the amount of 


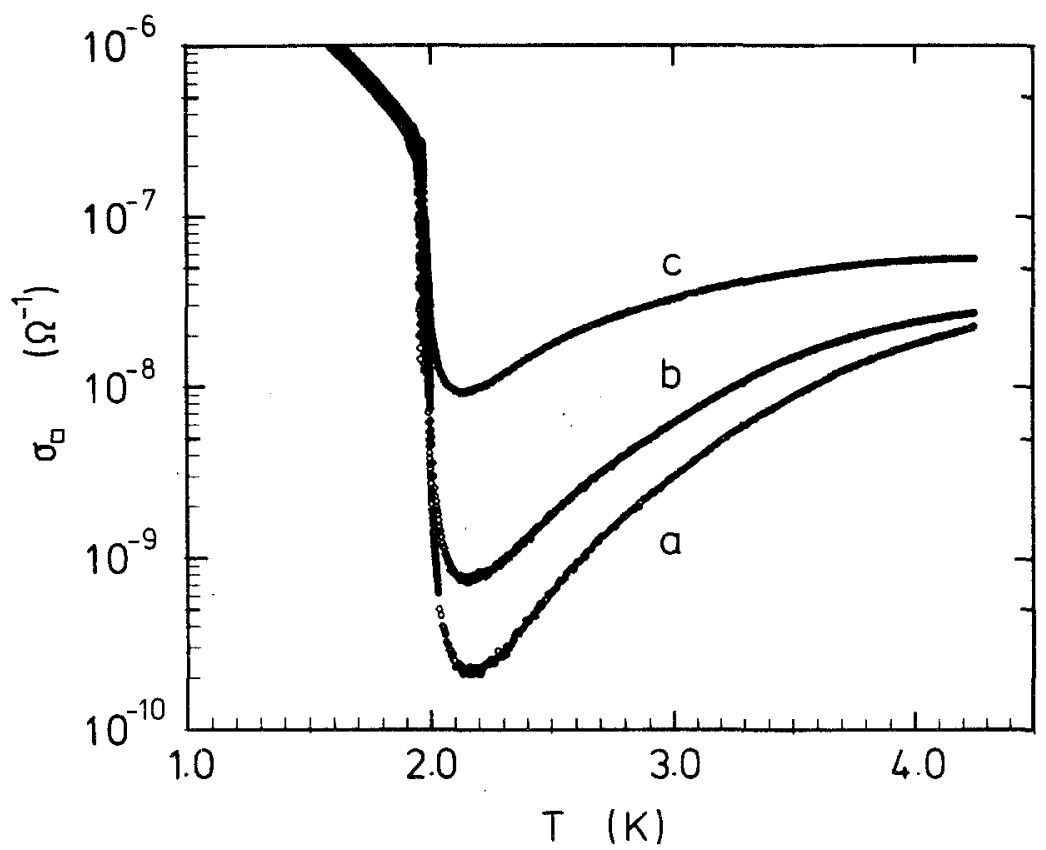

Fig. 5. Conductivity of SSE on hydrogen substrates annealed in three stages: (a) after annealing the film at $4.2 \mathrm{~K}$ for 1 hours; (b) after annealing at $4.2 \mathrm{~K}$ for 12 hours; (c) after annealing at $8 \mathrm{~K}$ for 1 hour. The curves correspond to Figs. $7 \mathrm{~b}-\mathrm{d}$ in $\mathrm{I}$. The shift of the temperature of the dip with respect to Fig. 3 is due to the larger amount of helium in the cell.

helium in the sample cell, were kept constant in these runs. As reported earlier, ${ }^{1}$ annealing increases the conductivity in the high temperature range, with the $\mathrm{H}_{2}$ substrate nearly bare, implying a reduction of the surface roughness. In the low temperature range the conductivity does not depend on the annealing history, as expected, if the saturated He film is thick enough to cover up the roughness of the hydrogen substrate. A plot of the transition regions on an expanded scale (Fig. 6) again clearly displays the conductivity dip. The data also give an impression of the reproducibility of the effect. A comparison of Figs. 6a-c shows that upon annealing the absolute value of the conductivity in the dip minimum increases, but the temperature of the minimum obviously does not change.

The variation of the signal with the amount of helium in the cell is shown in Fig. 7. The position of the dip closely follows the formation of the saturated helium film, which is shifted to lower temperature as helium is removed from the cell. According to the quartz gauge the film thickness where the minimum occurs remains unchanged. As seen from Fig. 7, also the bottom value of the dip depends very little on the amount of helium. 

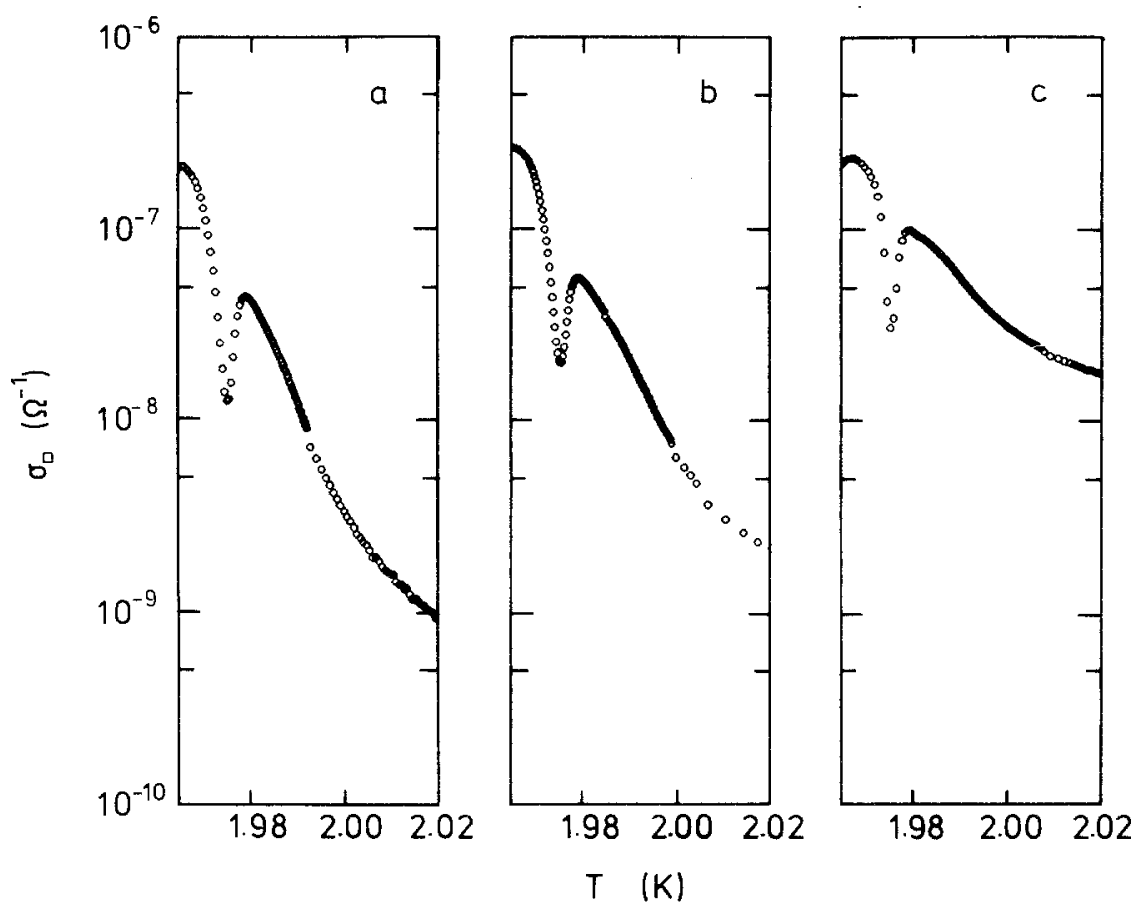

Fig. 6. Expanded view of Fig. 5. Note that the temperature of the dip is not affected by the annealing procedure.

\subsection{AC Driving Field}

Different frequencies between 1 and $100 \mathrm{kHz}$ have been used for the conductivity measurement. In this range the position and size of the dip turns out to be independent of the driving frequency. This fact excludes the possibility that the dip results from a resonance phenomenon, like a standing wave of third sound, etc. Likewise, the driving voltage was found to be irrelevant up to $1 \mathrm{~V}_{\mathrm{p}-\mathrm{p}}$.

\subsection{Charge Density and Holding Field}

These two quantities could not be varied independently, because upon increasing the electrical holding field $F_{z}$, which forces the SSE onto the film surface, residual electrons from the cell walls accumulated on the electrode segments, thereby increasing $n_{s}$. Thus it is still open whether the variation of the signal for different $d c$ voltages, as plotted against the quartz frequency in Figs. 8a-c, is mainly due to $n_{s}$ or $F_{z}$. For increasing holding 


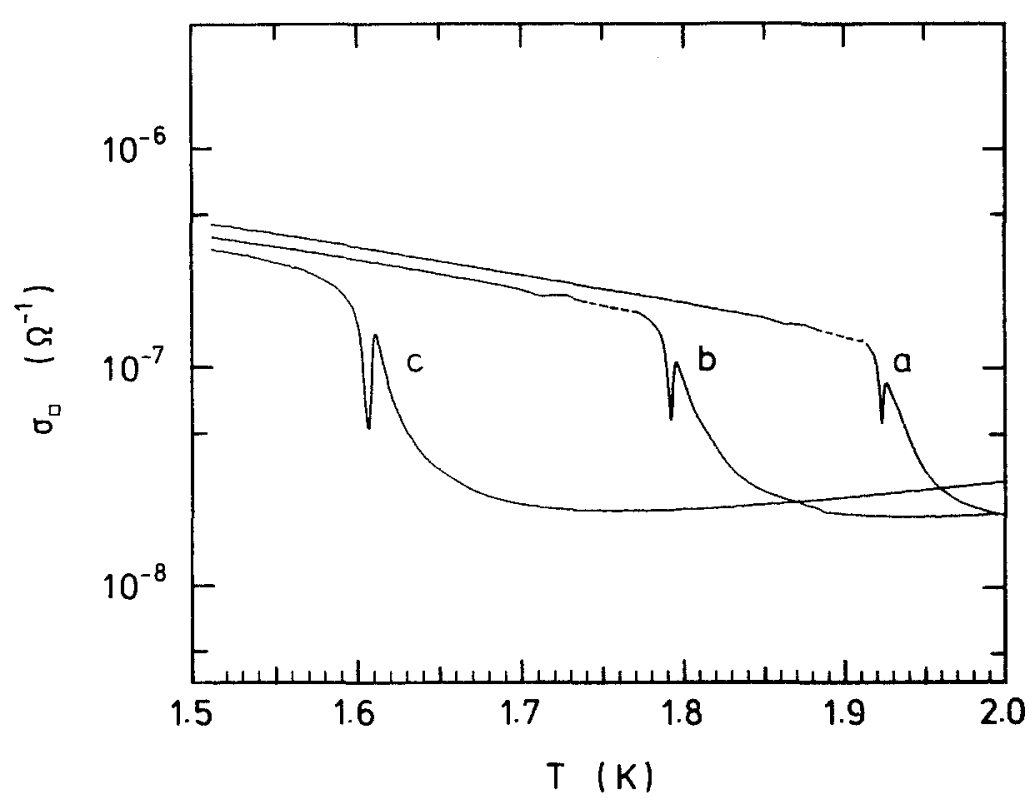

Fig. 7. Behavior of the conductivity dip as the amount of helium in the cell is reduced in steps from a to $c$. In the temperature intervals where the traces $b$ and $c$ are dashed a thermal instability in the cryostat prevented to take reliable data.

voltage a narrowing of the dip and a shift to smaller frequencies, i.e. smaller film thickness, is observed.

\section{DISCUSSION}

For a discussion of the results described in the previous section we first characterize the parameters of the conductivity dip in the following way (see Fig. 9): $\sigma_{1}$ is the conductivity extrapolated from low temperatures, where the SSE are located on a saturated helium film, up to the temperature $T_{d}$ of the dip minimum; $\sigma_{2}$ is the conductivity at $T_{d}$ obtained by straight interpolation of the behavior outside the dip; $\sigma_{3}$ is the actual conductivity in the dip minimum.

Instead of conductivities we henceforth use resistivities, which are better suited for the analysis, because contributions from various scattering processes-due to gas atoms, surface roughness and a mechanism yet to identify, leading to the dip-should be additive in the resistivity as long as they are independent. Thus we define $\rho_{1}, \rho_{2}$, and $\rho_{3}$ as the reciprocal values 


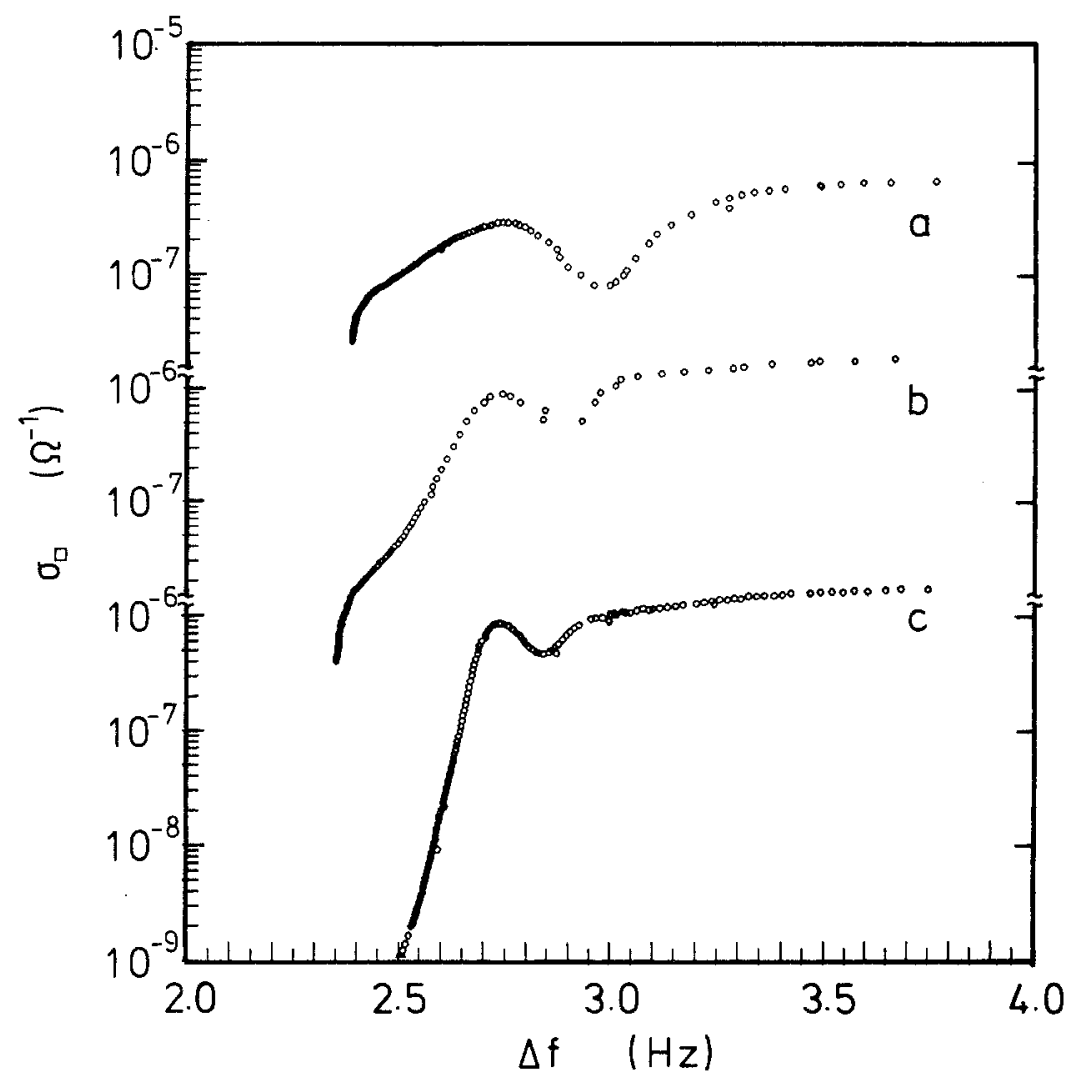

Fig. 8. SSE conductivity for various holding voltages plotted against the frequency of the quartz oscillator, a: $1 \mathrm{~V} ; \mathbf{b}: 10 \mathrm{~V} ; \mathbf{c}: 30 \mathrm{~V}$.

of $\sigma_{1}, \sigma_{2}$, and $\sigma_{3}$ introduced above. Results for various experimental conditions are summarized in Table $\mathrm{I}$.

The resistivity $\rho_{1}$ should be predominated by the electron scattering from helium gas atoms. As an additional test whether this is indeed the case on the saturated helium films in our temperature range, we have used a measurement of the magnetoresistance to determine the SSE mobility $\mu$, independently of the conductivity. ${ }^{*}$ Figure 10 shows the data, together with a theoretical result of Saitoh ${ }^{6}$ based on electron scattering from helium gas atoms. Although the experimental data are about a factor of 3 smaller, the

\footnotetext{
* The conductivity measured as described earlier is proportional to $\mu$, but also contains the surface electron density $n_{s}$, which may not be known very well. This is true in particular, if some fraction of the surface electrons are localized and do not contribute to $\sigma_{\square}$, as it is the case for rough hydrogen surfaces (see I).
} 


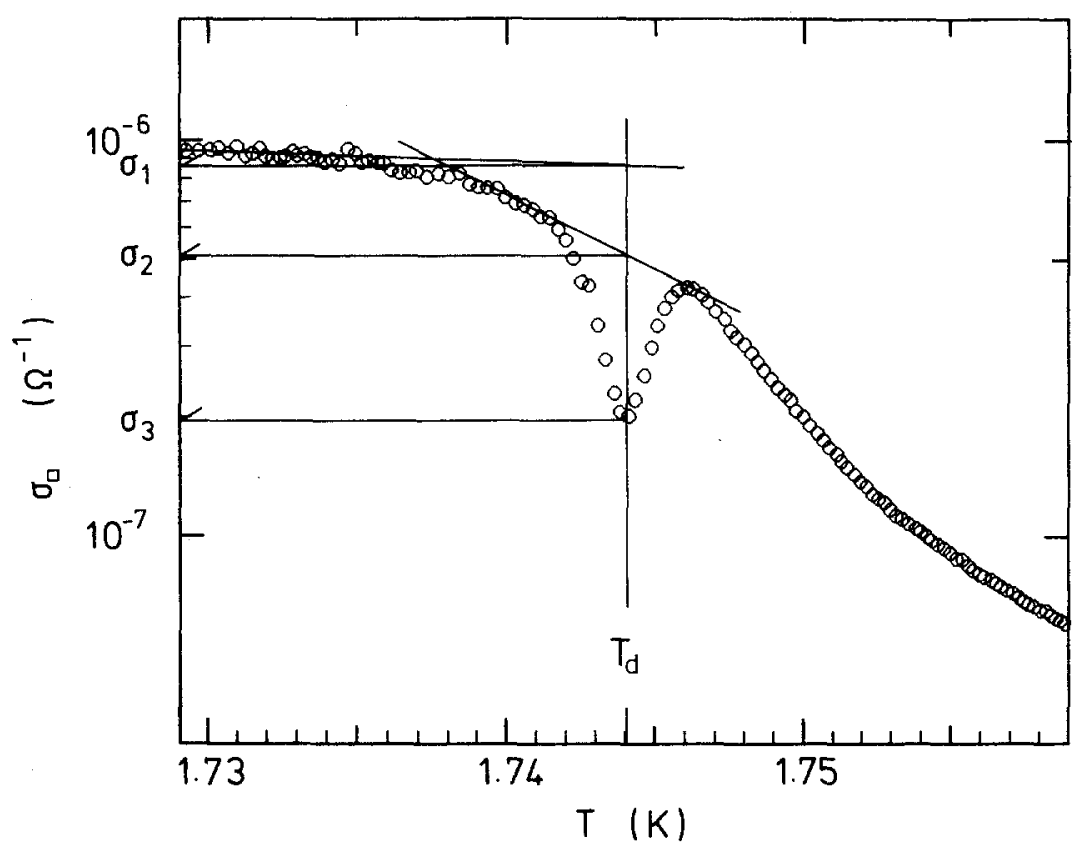

Fig. 9. Example for the determination of the conductivities $\sigma_{1}, \sigma_{2}$, and $\sigma_{3}$. For details, see text.

TABLE I

The Following Parameters Vary in the Different Groups: A: Surface Roughness; B, C: Amount of Helium in the Cell; D: Charge Density and Holding Field. $\rho_{1}, \rho_{2}$, and $\rho_{3}$ (in M $\Omega$ ). $T_{d}$ and (in K). $n_{s}\left(\right.$ in $10^{8} \mathrm{~cm}^{-2}$ )

\begin{tabular}{ccrrrrrr}
\hline Sample & $\rho_{1}$ & $\rho_{2}$ & \multicolumn{1}{c}{$\rho_{3}$} & $T_{d}$ & $E_{c}$ & $n_{s}$ & Symbol \\
\hline A-1 & 3.6 & 13.0 & 84.0 & 1.976 & 22.0 & 3.1 & $\bullet$ \\
A-2 & 3.2 & 9.8 & 52.0 & 1.976 & 18.0 & 3.1 & $\odot$ \\
A-3 & 4.0 & 6.8 & 34.0 & 1.976 & 11.0 & 3.1 & $\bigcirc$ \\
B-1 & 3.9 & 6.5 & 19.0 & 1.607 & 6.9 & 0.9 & $\square$ \\
B-2 & 5.8 & 8.5 & 18.0 & 1.792 & 6.9 & 0.9 & $\square$ \\
B-3 & 7.5 & 10.5 & 18.0 & 1.922 & 6.9 & 0.9 & $\square$ \\
C-1 & 0.71 & 1.7 & 5.2 & 1.665 & 11.0 & 7.2 & $\Delta$ \\
C-2 & 1.0 & 2.0 & 5.0 & 1.730 & 11.0 & 7.2 & $\triangle$ \\
C-3 & 1.7 & 2.7 & 5.5 & 1.835 & 11.0 & 7.2 & $\triangle$ \\
D-1 & 0.83 & 2.6 & 13.0 & 1.802 & 11.0 & 3.9 & $\nabla$ \\
D-2 & 0.48 & 1.0 & 2.0 & 1.802 & - & 13.0 & $\square$ \\
D-3 & 0.53 & 1.0 & 2.2 & 1.802 & 38.0 & 19.0 & $\nabla$ \\
\hline
\end{tabular}




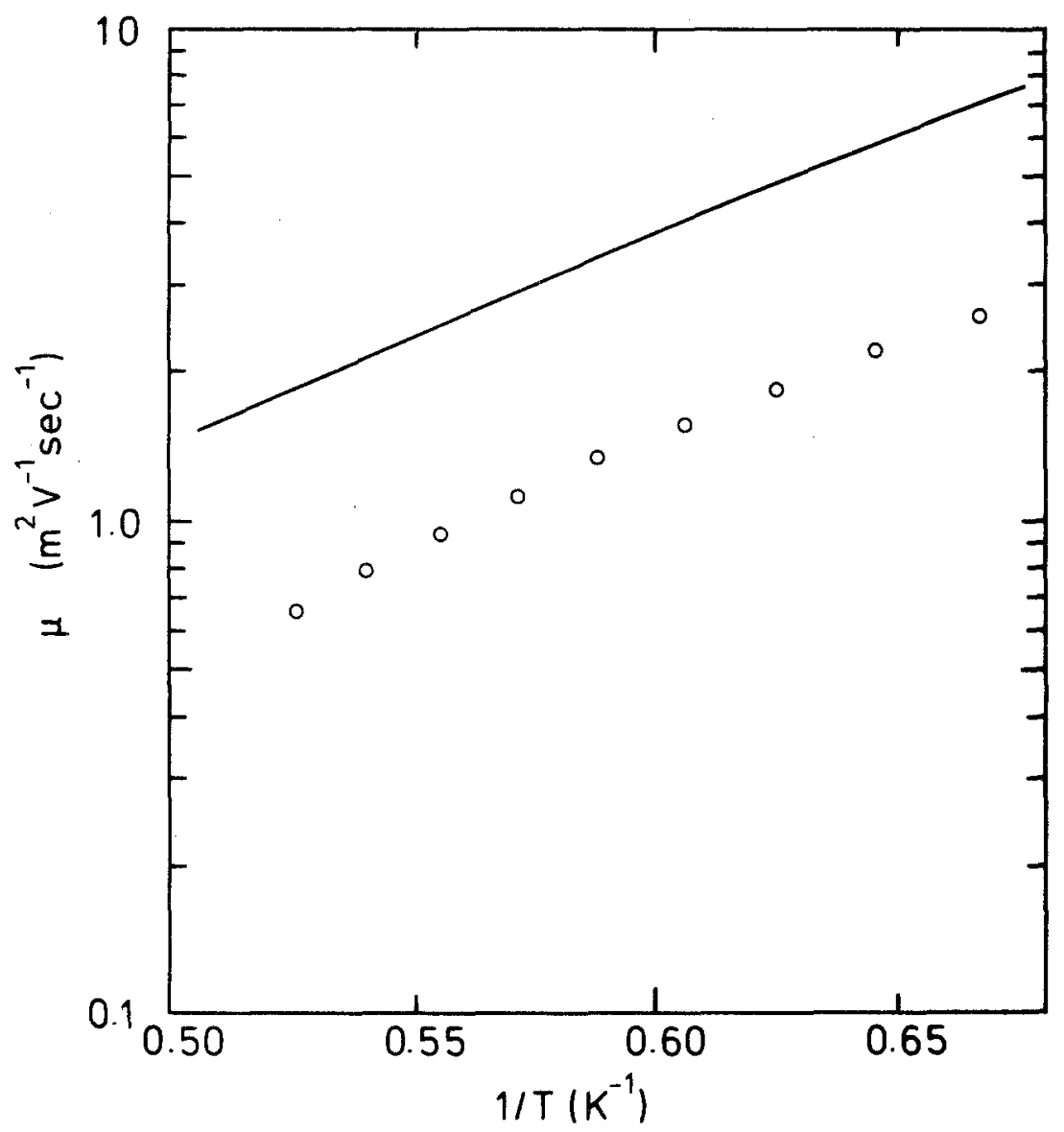

Fig. 10. Mobility on a saturated helium film obtained from a magnetoresistance measurement. The solid line is the theoretical result of Saitoh, in which the scattering from helium gas atoms was taken into account. The electron density $n_{s}$ was $3.9 \times 10^{8} \mathrm{~cm}^{-2}$.

temperature dependence is in very good agreement, supporting the assumption that gas atom scattering dominates for our saturated films.

The magnetoresistance measurement has also been used to determine the areal density of mobile electrons, $n_{s}$, an important parameter of the system, which is also listed in Table I.* It ought to be mentioned that a simple calculation of $n_{s}$ from the geometry of the electrodes and the applied

\footnotetext{
* Magnetoresistance measurements are sensitive only if the mobility is rather high, as on saturated helium films; unfortunately they were not applicable in the region of the unsaturated films, and in particular not near the conductivity dip.
} 
dc voltage proved to be unreliable. It was found that a certain amount of electrons on the order of some $10^{8} \mathrm{~cm}^{-2}$ was present on the surface due to stray fields, even if the dc voltage was zero; in order to reach smaller densities a negative bias voltage had to be applied.

The parameter $\rho_{2}$ in Table I can be identified with a fictitious "background resistivity" at the temperature $T_{d}$, interpolated smoothly from the overall behavior of the transition from high resistivity at small film thickness to a low resistivity state for thick films. Such a monotonous temperature dependence would be expected if the role of the growing helium film were only to lift the electrons further away from the surface, neglecting the scattering by ripplons on the helium film. (As shown earlier, the average distance of the SSE above solid $\mathrm{H}_{2}$ is $1.7 \mathrm{~nm}$, whereas on bulk liquid ${ }^{4} \mathrm{He}$ it is $7.6 \mathrm{~nm}$.) In the high resistivity region electron localization is probably important. ${ }^{9,10}$ As the distance of the electrons from the surface is increased, scattering by surface roughness becomes less effective. Moreover, the roughness of the $\mathrm{H}_{2}$ surface is smeared out the thicker the liquid He film. Both effects give rise to a monotonous decrease of the resistivity with growing film thickness. We would like to add that we have no indication that for our system the high resistivity at small film thickness should be associated with the formation of a polaron state, as suggested for SSE on helium films above a sapphire substrate. ${ }^{4}$ In this simple picture then the background resistivity $\rho_{2}$ at $T_{\dot{c}}$ consists of the two contributions due to gas atom scattering $\left(=\rho_{1}\right)$ and scattering from surface roughness $\left(=\rho_{2}-\rho_{1}\right)$.

The data listed in Table $I$ are grouped in 4 categories:

1. Group A corresponds to Fig. 6, for which the amount of helium inside the cell and $n_{s}$ were fixed, while the degree of annealing was changed. Accordingly also the degree of surface roughness decreased from A-1 to A-3, reflected in the decreasing activation energy $E_{c}$.

2. Group B corresponds to Fig. 7. The amount of helium in the cell was reduced from B-1 to B-3, leading to a shift of the position of the dip with respect to temperature $\left(T_{d}\right)$. The degree of annealing and $n_{s}$ were kept constant.

3. Group $C$ is similar to $B$, except that the electron density was a factor of 8 larger in this case $\left(7.2 \times 10^{8} \mathrm{~cm}^{-2}\right.$ instead of $\left.0.9 \times 10^{8} \mathrm{~cm}^{-2}\right)$.

4. In group $\mathrm{D}$ the electron density $\boldsymbol{n}_{s}$ was the quantity to be varied, while the other parameters were kept constant, corresponding to Fig. 8.

For the error of the resistivities given in Table I we estimate less than $10 \%$ for $\rho_{2}$ and $\rho_{3}$, and possibly somewhat more for $\rho_{1}$.

The following features of the resistivity peak (corresponding to the conductivity dip) at $T_{d}$ can be derived from Table I:

1. As shown by group $A$, the value of the peak maximum, $\rho_{3}$, as well as the relative peak height, $\rho_{3}-\rho_{2}$, decrease as the surface roughness is 
reduced by annealing. The gas atom contribution $\rho_{1}$ is essentially constant, because $T_{d}$ does not change in this case.

2. A variation of $T_{d}$ by the amount of helium, given in groups $\mathrm{B}$ and C, leads to a change in $\rho_{1}$, as expected. However, $\rho_{3}$ is nearly constant in these groups and so is $\rho_{2}-\rho_{1}$.

3. An increase of the electron density (group D) leads to a decrease of both the absolute and the relative peak height.

In addition to these trends there is a strikingly strong correlation between the values of $\rho_{3}$ and $\rho_{2}-\rho_{1}$. This is illustrated in Fig. 11, where these two quantities are plotted for all the runs of Table I. The resulting dependence roughly implies a proportionality between $\rho_{3}$ and $\rho_{2}-\rho_{1}$.

We now turn to a discussion of possible contributions of the observed phenomena. First, the role of correlations between the electrons should be

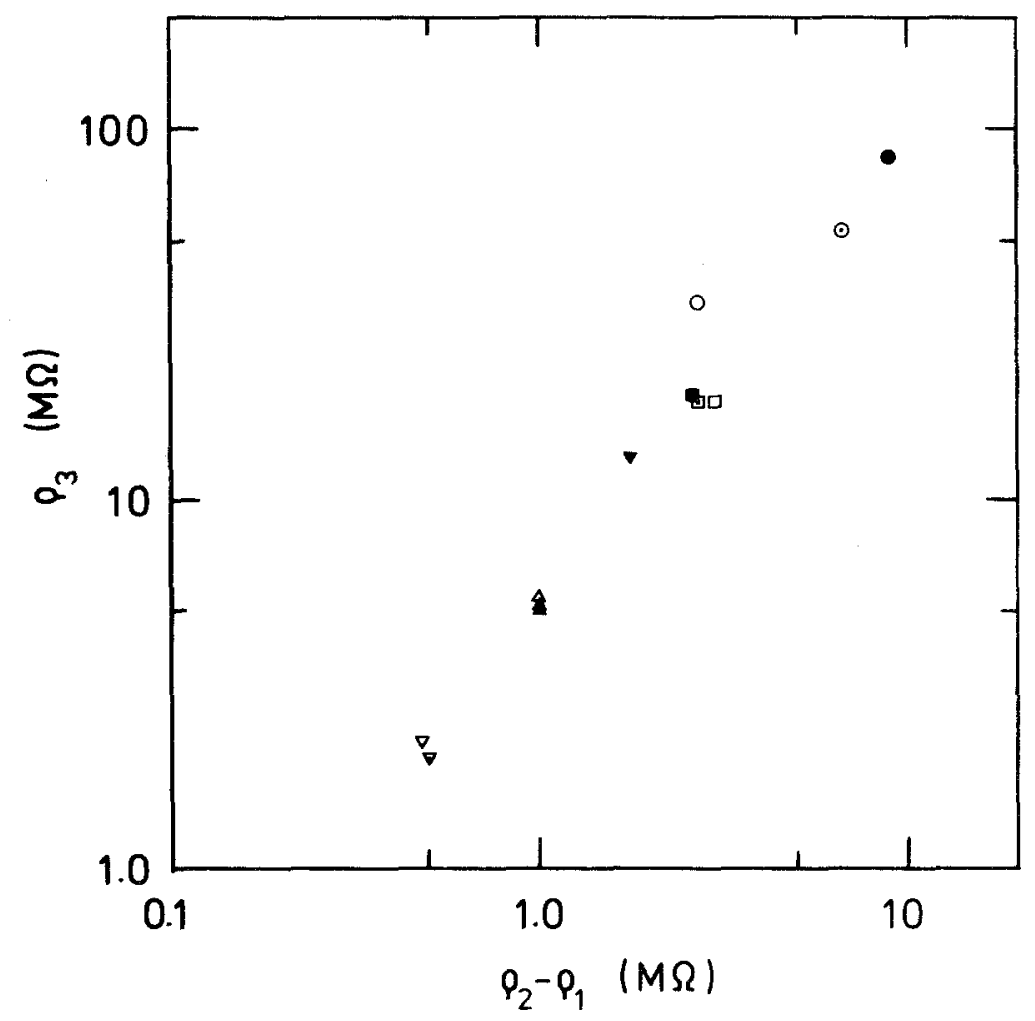

Fig. 11. Values of the resistivity peak $\rho_{3}$ against $\rho_{2}-\rho_{1}$, taken from Table I. Although there are several factors ( $n_{s}$, surface roughness, density of helium gas atoms) which contribute to the resistivity, a clear correlation is observed between $\rho_{3}$ and $\rho_{2}-\rho_{1}$. Symbols are defined in Table $\mathbf{I}$. 
clarified. It is known that at low temperature a classical two-dimensional gas of electrons, as it is formed by the SSE on helium, undergoes a phase transition to a so-called Wigner crystal. The mobility in this state can be low, because pinning of only a few electrons will be sufficient to restrict the motion of the rest. In addition, in the Wigner state one expects a dimple in the helium film to form underneath each electron, because the electrons are essentially localized on lattice positions and, in contrast to the gas state, do not move across the surface with a speed much larger than the velocity of capillary waves.

Such an influence of correlations between the electrons was in fact observed in our measurements at high electron densities. In Fig. 12, the resistivity $\rho_{\text {svp }}(1.5 \mathrm{~K})$ for electrons on a saturated helium film at $1.5 \mathrm{~K}$ is plotted against the electron density. Upon increasing $n_{s}$, the resistivity first

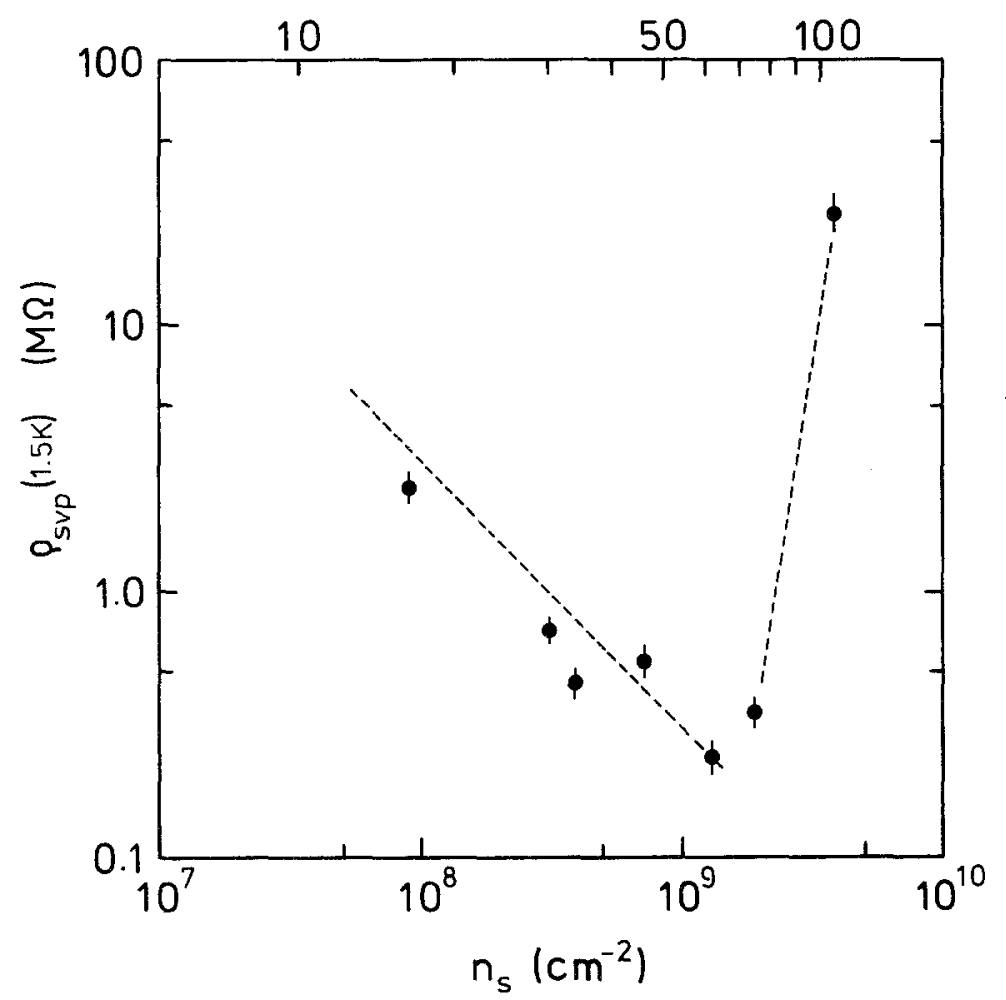

Fig. 12. $n_{s}$ dependence of the resistivity on a saturated helium film at a temperature of $1.5 \mathrm{~K}$. The dashed line is a guide to the eye. 
drops due to the larger amount of charge carriers contributing to the $a c$ current. At $n_{s}=4 \times 10^{9} \mathrm{~cm}^{-2}$, however, steep increase in $\rho_{s v p}$ appears. Expressed in terms of the dimensionless parameter $\Gamma=e^{2} n_{s}^{-1 / 2} / k_{B} T$, the ratio between Coulomb energy and thermal energy of the SSE, this increase is observed slightly above $\Gamma=10^{2}$, close to the value $\Gamma=130$ where Wigner crystallization is known to occur on bulk helium. ${ }^{11}$ We therefore associate the steep rise in resistivity at large $n_{s}$ with increasing correlations of the 2D electron system near solidification. This implies, on the other hand, that correlation effects should be of minor importance in the region where the conductivity dip is observed, because in the examples listed in Table I the temperature in general is higher and the electron density lower than above, and consequently the value of $\Gamma$ is distinctly smaller than $10^{2}$.

Next we consider the explanation of PI, based on electron-ripplon scattering, which has already been introduced in Sec. 2. Figure 13 shows the conductivity calculated according to this model from Eqs. (2)-(4). Although at $1 \mathrm{~K}$ the conductivity dip predicted by this ripplon mechanism

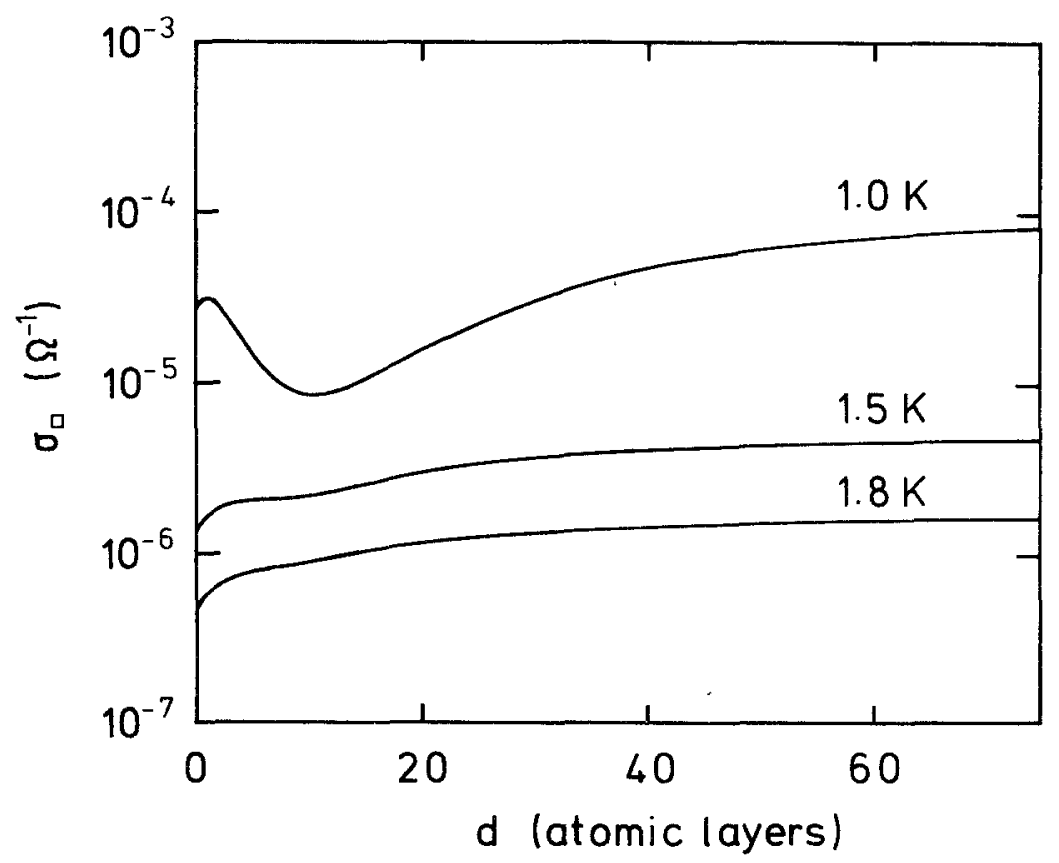

Fig. 13. SSE conductivity calculated for scattering from helium gas atoms and ripplons (Eqs. (3) and (4)) as a function of the helium film thickness $d$. The electron density is taken to be $n_{s}=4 \times 10^{-2}$. 
is quite pronounced, it is hardly discernible at $1.5 \mathrm{~K}$ and $1.8 \mathrm{~K}$, because gas atom scattering overshadows the ripplon scattering at these higher temperatures. Since our measurements were done at $T>1.6 \mathrm{~K}$, the ripplon mechanism alone therefore appears not to be sufficient to account for the experimentally observed well-developed dips.

In the calculation of PI, the dip is much more prominent than in Fig. 13, even at relatively high temperature (cf. Fig. 4 in PI). It has to be pointed out, though, that in these calculations instead of the integral over the electron wave function (Eq. (4)) an average distance $\langle z\rangle$ of the electrons from the helium surface has been used. The scattering rate of electrons from ripplons resulting from the full expression, Eq. (4), is nearly an order of magnitude smaller than in the simplified treatment, and therefore the dip here is less pronounced.

Additional features at variance with the simple ripplon mechanism are the width and relative height of the resistivity peak. For a mechanism which is just additive to the other scattering processes-as expected for ripplon scattering-the relative peak height ought to be unchanged as long as $\boldsymbol{n}_{\boldsymbol{s}}$ is being kept constant, whatever the variation of $\sigma_{2}$. This is in conflict with groups $\mathrm{A}, \mathrm{B}$, and $\mathrm{C}$ in Table $\mathrm{I}$, where clear variations of the relative peak height $\rho_{3}-\rho_{2}$ are observed. The width of the peak, although possibly not too well characterized by our thickness gauge, nevertheless appears to be distinctly narrower than predicted by the ripplon mechanism, even if the latter is considered at low temperature (cf. Figs. 8 and 13).

Finally, we discuss a mechanism based on the ripplonic polaron or dimple formation. There is a lot of work on ripplonic polarons. ${ }^{12-16}$ These theories commonly yield the result that the energy gain due to polaron formation is so small that a stable polaron cannot exist in the temperature range above $1 \mathrm{~K}$ under consideration here. Moreover, the coupling constant which controls the polaron formation is small on a thin He film, and grows monotonously with increasing film thickness. Thus there is no maximum of the coupling constant as a function of film thickness which one could correlate with the resistivity peak. This is due to the way how the cutoff length is chosen, which usually is taken to be the capillary length. There are some doubts about this choice, however, ${ }^{4,17}$ Andrei, for example, has suggested to take the mean electron separation as cutoff length. On a rough surface the effect of localization may well play a role to restrict this length scale.

A rough estimate, which shows that there should be indeed a tendency of the electrons to form a dimple state in some preferential thickness range, is obtained from the following simple model: Consider the dimple as a nearly spherical indentation in the film surface with depth $\Delta z$ and size $r_{0}$, where $r_{0}$ is taken to be the thermal de Broglie wavelength of the electron. 
The formation energy of such a dimple state is then given by

$$
\Delta E(\Delta z)=-e F_{z} \Delta z+\sigma \pi \Delta z^{2}+g^{\prime} \rho\left(\frac{\pi}{2} r_{0}^{2} \Delta z\right)\left(\frac{\Delta z}{3}\right)
$$

Here $\sigma$ is again the surface tension of liquid helium and $g^{\prime}$ is an effective van der Waals acceleration, which for a thin film is much larger than the acceleration due to gravity. The first term on the rhs of Eq. (7) represents the electrostatic energy of the electrons in the electric field $F_{z}$ given by Eq. (4). The second term results from the increase of the surface area on forming the dimple, and the last one is due to the van der Waals force. The equilibrium deformation $\Delta z_{0}$ is determined by minimizing the energy, Eq. (7). Figure 14 shows the energy of formation as a function of the helium film thickness. The maximum energy gain is about $0.1 \mathrm{~K}$, an order of magnitude smaller than the ambient temperature. Therefore a stable dimple will not exist. Nevertheless the system is close to being unstable, and there will be a tendency for transient dimple or polaron formation, which according to Fig. 14 is expected to be most pronounced around the fi!m thickness of the maximum energy gain at about 10 monolayers.

Since an electron-dimple complex should strongly interact with surface roughness, the resistivity in this state will be a function of $\rho_{2}-\rho_{1}$ (which,

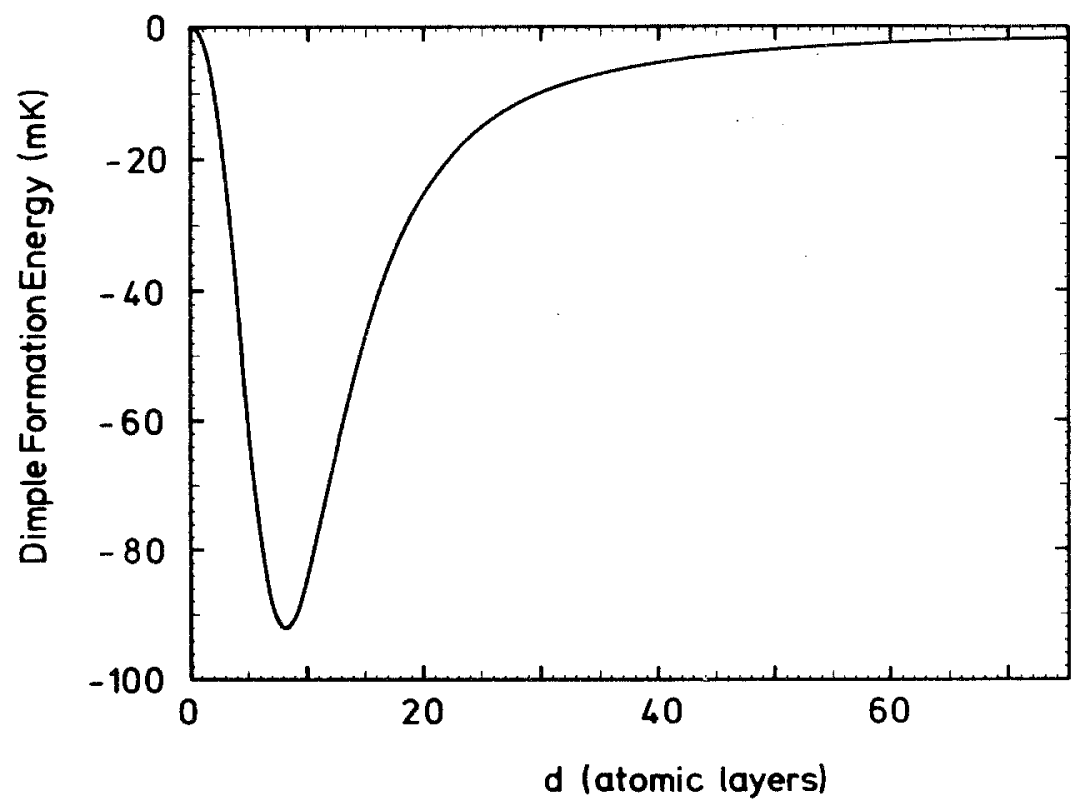

Fig. 14. Net formation energy of a dimple, derived from the model given by Eq. (7), as a function of the helium film thickness. The electron density is taken to be $4 \times 10^{8} \mathrm{~cm}^{-2}$. 
as shown earlier, gives a measure for the surface roughness). This is in agreement with the correlation found in Fig. 11. Another aspect of the polaron picture in accordance with the data in Table I is that the resistivity due to the formation of an electron-dimple complex will not simply be additive to $\rho_{2}$ (i.e. the relative peak height in group B and C is not expected to be constant), because $\rho_{2}$ is extrapolated to $T_{d}$ under the assumption of a different state, namely free electrons.

It should be noted that Saitoh's mass formula ${ }^{14}$ of a strongly coupled polaron has a similar dependence on the He film thickness, although the present experimental condition is not fully compatible with the validity limit of the theory.

\section{SUMMARY}

We have investigated a conductivity dip of the surface state electrons on a quench condensed hydrogen film surface. The dip appears when about 10 atomic layers of helium are adsorbed onto the hydrogen surface. It depends on the degree of the surface roughness and also on the electron density $n_{s}$ and/or the applied electric field. The other experimental parameters, like temperature, and driving frequency and voltage of the ac conductivity measurement were found to be irrelevant for the phenomenon.

Electron scattering from ripplons and a ripplonic polaron have been considered as possible candidates for the interpretation of the conductivity dip. It is found that scattering from ripplons alone as treated by Paalanen abd Iye in order to explain the phenomenon is not sufficient to interpret the present experimental results. Likewise, assuming the formation of a ripplonic polaron does not completely account for the data because the energy gained by forming the polaron state is estimated to be an order of magnitude smaller than the thermal energy in our experiment, and therefore a stable polaron should not exist. It is argued, however, that the electrons, being close to a polaron instability, could encounter a reduction in mobility due to the formation of transient dimples. Certain features of the polaron picture are in qualitative agreement with the observations. For a quantitative understanding of the phenomenon obviously a more complete theoretical treatment is required, which takes the various aspects of the electron-film interactions into account, as well as electron-electron correlations and localization effects. We hope that the dependences investigated here can serve as a guideline for these theoretical efforts.

\section{ACKNOWLEDGMENTS}

The major part of this work was carried out at the Johannes-GutenbergUniversity Mainz, FRG. One of us (K.K.) appreciates financial support 
from Mombusyou and Alexander von Humboldt-Stiftung. Helpful discussion with M. Saitoh, V. B. Shikin, and A. J. Dahm are gratefully acknowledged.

\section{REFERENCES}

1. K. Kono, U. Albrecht, and P. Leiderer, J. Low Temp. Phys. 82, 279 (1991).

2. M. A. Paalanen and X. Iye, Phys. Rev. Lett. 55, 1761 (1985).

3. D. Cieslikowski, A. J. Dahm, and P. Leiderer, Phys. Rev. Lett. 58, 1751 (1987).

4. E. Y. Andrei, Phys. Rev. Lett. 52, 1449 (1984).

5. K. Kajita and W. Sasaki, Surf. Sci. 113, 419 (1982).

6. M. Saitoh, J. Phys. Soc. Jpn. 42, 201 (1977).

7. W. T. Sommer and D. J. Tanner, Phys. Rev. Lett. 20, 1345 (1976).

8. E. Cheng and M. W. Cole, Phys. Rev. B 38987 (1988).

9. P. W. Adams and M. A. Paalanen, Phys. Rev. Lett. 58, 2106 (1987).

10. P. W. Adams and M. A. Paalanen, Phys. Rev. Lett. 61, 451 (1988).

11. C. C. Grimes and G. Adams, Phys. Rev. Lett. 42, 795 (1979).

12. Yu. P. Monarkha, Fiz. Nizk. Temp. 1, 526 (1975). [Sov. J. Low Temp. Phys. 1, 258 (1975).]

13. S. A. Jackson and P. M. Platzman, Phys. Rev. B 24, 499 (1981).

14. M. Saitoh, J. Phys. C 16, 6995 (1983).

15. M. H. Degani and O. Hipolito, Phys. Rev, B 32, 3300 (1985).

16. F. M. Peeters and S. A. Jackson, Phys. Rev. B 341539 (1986).

17. S. A. Jackson and P. M. Platzman, Surf. Sci. 142, 125 (1984). 